\title{
Flexible Resource Allocation for Joint Optimization of Energy and Spectral Efficiency in OFDMA Multi-Cell Networks
}

\author{
Wenpeng Jing, Zhaoming Lu, Xiangming Wen, Zhiqun Hu, and Shaoshi Yang
}

\begin{abstract}
The radio resource allocation problem is studied, aiming to jointly optimize the energy efficiency (EE) and spectral efficiency (SE) of downlink OFDMA multi-cell networks. Different from existing works on either EE or SE optimization, a novel EE-SE tradeoff (EST) metric, which can capture both the EST relation and the individual cells' preferences for $\mathrm{EE}$ or SE performance, is introduced as the utility function for each base station (BS). Then the joint EE-SE optimization problem is formulated, and an iterative subchannel allocation and power allocation algorithm is proposed. Numerical results show that the proposed algorithm can exploit the EST relation flexibly and optimize the EE and SE simultaneously to meet diverse EE and SE preferences of individual cells.
\end{abstract}

Index Terms-Energy efficiency, joint optimization, OFDMA multi-cell, resource allocation, spectral efficiency.

\section{INTRODUCTION}

$\mathbf{S}$ PECTRAL efficiency (SE) has long been an important metric in wireless communication system design due to the scarcity of the spectrum resource and the consistently growing demands of wireless data traffic. On the other hand, energy efficiency (EE) in wireless networks has been attracting great interests recently owning to environmental and financial considerations. Since radio resource allocation has a great impact on the performance of OFDMA networks, the EE or SE optimization based on dynamic resource allocation has been studied extensively [1]-[5]. However, EE and SE do not always coincide with each other, and sometimes the two metrics may even conflict [6]. Hence, how to balance the EE and SE remains a problem worth studying.

In [6], the EE-SE tradeoff (EST) relation in single cell OFDMA network is investigated and a low-complexity resource allocation algorithm that can balance the EE and SE is proposed. In [7], the EST problem in interference-limited wireless networks is investigated and a power allocation algorithm is devised. The EST optimization problem in the distributed antenna systems (DAS) with and without fairness consideration is investigated in [8] and [9], respectively, and the corresponding optimal power allocation algorithms are developed. However, all these works formulate the EST problem as an EE optimization problem, while SE is considered as a constraint. Although a

Manuscript received July 24, 2014; revised December 26, 2014; accepted Janaury 5, 2015. This work was supported by the National Natural Science Foundation of China (61271179). The associate editor coordinating the review of this paper and approving it for publication was D. W. K. Ng.

W. Jing, Z. Lu, X. Wen, and Z. Hu are with the School of Information and Communication Engineering, Beijing University of Posts and Telecommunications, Beijing 10086, China (e-mail: jingwenpeng@bupt.edu.cn; 1zy_0372@ 163.com; hn208@163.com; huzhiqun@bupt.edu.cn).

S. Yang is with the School of Electronics and Computer Science, University of Southampton, Southampton SO17 1BJ, U.K. (e-mail: sy7g09@ecs. soton.ac.uk).

Digital Object Identifier 10.1109/LCOMM.2015.2392113 unified EST metric for point-to-point links has been proposed in the recent work [10], the single wireless channel scenario considered is less pragmatic compared to the multi-user scenario, hence the proposed optimal power control scheme cannot be directly used in the practical cellular networks. Currently, studies on radio resource allocation in multi-cell OFDMA networks still focus on the conventional SE-based [1] or EE-based [3] optimization frameworks, while the EST-based works are limited. Note that multi-cell cellular networks experience fluctuant demands on data traffic in both time and space, and every base station (BS) has divergent availability of energy and spectral resources. The dynamic radio resource supply and demand differences among BSs result in that individual cells' preferences for EE and SE performance differ from each other. Hence, the existing EE-optimal and SE-optimal methods, or even the EST-based algorithms, are not directly applicable to the multicell scenarios, and it remains an open problem to tradeoff and optimize the EE and SE performance jointly and flexibly.

The main contributions of this letter are twofold. Firstly, the joint optimization of the EE and SE is investigated, considering the different EE-SE preferences at BSs in downlink OFDMA multi-cell networks. Secondly, a coordinated radio resource allocation algorithm is proposed, which can allocate the power and subchannels iteratively and can optimize the utilization of the energy and spectral resources simultaneously.

\section{System Model and Problem Formulation}

\section{A. System Model}

We consider the downlink transmission in a cluster of coordinated BSs. The following parameters are used to describe the system model:

- $\mathcal{M}$ : BS set and $\mathcal{M}=\{1,2, \cdots, M\}$.

- $\mathcal{K}_{m}$ : User set who connected to BS $m$ and $\mathcal{K}_{m}=$ $\left\{1,2, \cdots, K_{m}\right\}$.

- $\mathcal{N}$ : Subchannel set and $\mathcal{N}=\{1,2, \cdots, N\}$.

- $B$ : The bandwidth of each subchannel.

- $g_{m, k}^{n}$ : The gain of subchannel $n$ from BS $m$ to user $k$.

- $p_{m}^{n}$ : The transmit power of BS $m$ on subchannel $n$.

- $s_{m, k}^{n}$ : The subchannel allocation indicator indicating whether or not subchannel $n$ is allocated to user $k$ of $\mathrm{BS}$ $m$, and $s_{m, k}^{n}=\{0,1\}$.

Perfect channel state information (CSI) is assumed available to both receivers and transmitters. In FDD systems, this is achieved by users' estimation and feedback, while in TDD systems, the CSI is estimated at the BS and then used for downlink transmission due to the channel reciprocity. The data rate of user $k$ in cell $m$ on subchannel $n$ may be expressed as $r_{m, k}^{n}=B \log _{2}\left(1+\frac{p_{m}^{n} g_{m, k}^{n}}{I_{m, k}^{n}+\sigma^{2}}\right)$, where $I_{m, k}^{n}=\sum_{j \in \mathcal{M} \backslash\{m\}} p_{j}^{n} g_{j, k}^{n}$ denotes the power of inter-cell interference, and $\sigma^{2}$ represents 
the power of the additive white Gaussian noise imposed on each subchannel. Then, the aggregate rate for user $k$ and the total throughput of BS $m$ are given by $R_{m, k}=\sum_{n \in \mathcal{N}} s_{m, k}^{n} r_{m, k}^{n}$ and $R_{m}=\sum_{k \in \mathcal{K}_{m}} \sum_{n \in \mathcal{N}} s_{m, k}^{n} r_{m, k}^{n}$, respectively. The total power consumption of BS $m$ can be denoted as $P_{m}^{t o t}=\xi \sum_{n \in \mathcal{N}} p_{m}^{n}+p_{c}$, where $\xi$ is a constant power-amplifier inefficiency factor of the transmitter and $p_{c}$ is a constant denoting the circuit power consumption. Then, the SE and EE of BS $m$ can be described as

$$
\eta_{m}^{S E}=\frac{R_{m}}{\sum_{k \in \mathcal{K}_{m}} \sum_{n \in \mathcal{N}} s_{m, k}^{n} B} \quad \text { and } \quad \eta_{m}^{E E}=\frac{R_{m}}{P_{m}^{t o t}},
$$

respectively.

\section{B. Problem Formulation}

Our target is to exploit the EST relation and optimize BSs' EE and SE performance jointly. A novel EST metric, which is defined as

$$
U_{m}=\left(\eta_{m}^{S E}\right)^{w_{m}}\left(\eta_{m}^{E E}\right)^{1-w_{m}},
$$

is introduced to be the utility function for each BS. In (2), $w_{m}$ is the preference factor of BS $m$ and has the value range of $[0,1]$. The specific value of $w_{m}$ may be set by the operator based on the importance of the SE and EE. More specifically, if values of $w_{m}$ are closer to $1, \mathrm{BS} m$ prefers to maximize the SE; otherwise, improving the EE is given higher priority. Hence, the EST metric (2) is able to capture the EST relation in a unified way, thus, providing an effective representation of the composite utility that BSs can achieve. Note that the EST metric (2) proposed in this letter is designed for the OFDMA multi-user scenario and takes both the power and subchannel allocation into consideration. This is different from the work [10], which only focus on the power allocation to optimize the EST performance in the single channel scenario.

The geometric-mean instead of the arithmetic-mean of all BSs' utilities is considered, in order to make the problem more tractable and to provide the utility fairness amongst multiple cells [4]. Hence, the utility of the entire network is formulated as $U=\sum_{m \in \mathcal{M}} \log _{2}\left(U_{m}\right)$ and the joint optimization problem of $\mathrm{EE}$ and $\mathrm{SE}$ in the multi-cell cellular networks can be formulated as follows,

$$
\begin{aligned}
& \max _{\left\{s_{m, k}^{n}\right\},\left\{p_{m}^{n}\right\}} \sum_{m \in \mathcal{M}} \log _{2}\left(U_{m}\right) \\
& \text { s.t. } \quad \sum_{n \in \mathcal{N}} p_{m}^{n} \leq P_{m}^{\max }, \forall m \in \mathcal{M} \text {, } \\
& \sum_{k \in \mathcal{K}_{m}} s_{m, k}^{n} \leq 1, \forall n \in \mathcal{N}, \forall m \in \mathcal{M} \text {, } \\
& s_{m, k}^{n} \in\{0,1\}, p_{m}^{n} \geqslant 0, \forall n \in \mathcal{N}, \forall k \in \mathcal{K}_{m}, \forall m \in \mathcal{M},
\end{aligned}
$$

where the constraint ( $3 \mathrm{~b}$ ) limits the maximum transmit power of each BS, and the constraint (3c) implies that each subchannel can only be allocated to at most one user in each cell.

\section{Subchannel Allocation And Power Allocation Scheme}

The optimization problem (3) is a non-convex mixed binary integer programming problem, which is computationally prohibitive to find the optimal solution. Thus, we divide it

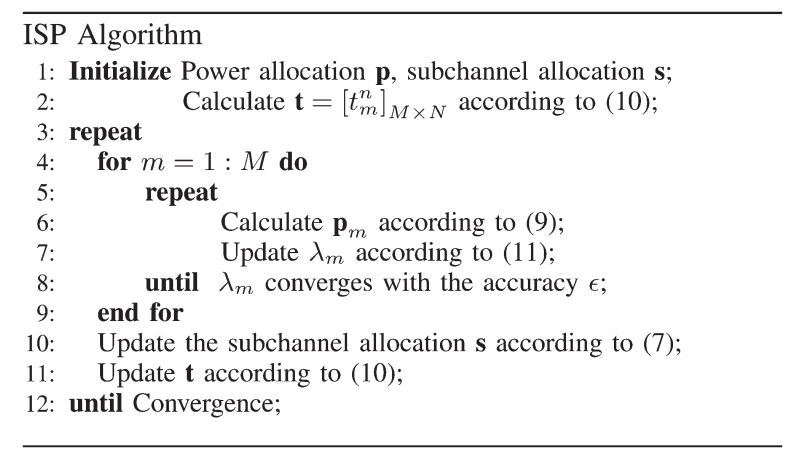

Fig. 1. ISP Algorithm.

into two subproblems, and devise an algorithm that carries out the subchannel allocation and power allocation separately and iteratively.

\section{A. Subchannel Allocation Strategy for a Given Power Allocation}

During the subchannel allocation, the total available spectrum is assumed to be occupied by each BS, and all the available subchannels will be allocated to the users served by each BS, which means $\sum_{k \in \mathcal{K}_{m}} \sum_{n \in \mathcal{N}} s_{m, k}^{n}=N$. Given a feasible power allocation matrix $\mathbf{p}=\left[\mathbf{p}_{m}\right]_{M \times 1}=\left[p_{m}^{n}\right]_{M \times N}$, the problem (3) is simplified into a subchannel allocation problem expressed by

$$
\max _{\left\{s_{m, k}^{n}\right\}} \sum_{m \in \mathcal{M}}\left\{\log _{2}\left[R_{m}(\mathbf{p})\right]+C_{m}(\mathbf{p})\right\} \quad \text { s.t. }(3 \mathrm{c})(3 \mathrm{~d}),
$$

where $C_{m}(\mathbf{p})=-\left(1-w_{m}\right) \log _{2}\left(P_{m}^{t o t}\right)-w_{m} \log _{2}(N B)$ is invariable when the power allocation matrix $\mathbf{p}$ has been given.

Lemma 1: The optimal solution of the problem (4) is achieved when

$$
s_{m, k}^{n}= \begin{cases}1, & \text { if } k=\arg \max _{k^{\prime} \in \mathcal{K}_{m}} r_{m, k^{\prime}}^{n}(\mathbf{p}), \\ 0, & \text { otherwise. }\end{cases}
$$

Proof: Note that when the power allocation matrix $\mathbf{p}$ has been given, i.e., $p_{m}^{n}$ for all $n \in \mathcal{N}, m \in \mathcal{M}$ has been fixed, the overall SINR of all users in each subchannel is also fixed, which is not affected by the other BSs' subchannel allocation. Hence, the problem (4) can be decomposed into $M$ subproblems, i.e.,

$$
\begin{aligned}
& \max _{\left\{s_{m, k}^{n}\right\}} \sum_{m \in \mathcal{M}}\left\{\log _{2}\left[R_{m}(\mathbf{p})\right]+C_{m}(\mathbf{p})\right\} \\
& =\sum_{m \in \mathcal{M}}\left\{\max _{\left\{s_{m, k}^{n}\right\}} \log _{2}\left[R_{m}(\mathbf{p})\right]\right\}+\sum_{m \in \mathcal{M}} C_{m}(\mathbf{p}) \\
& =\sum_{m \in \mathcal{M}}\left\{\log _{2}\left[\max _{\left\{s_{m, k}^{n}\right\}} R_{m}(\mathbf{p})\right]\right\}+\sum_{m \in \mathcal{M}} C_{m}(\mathbf{p}) .
\end{aligned}
$$

For each subproblem $\max _{\left\{s_{m, k}^{n}\right\}} R_{m}(\mathbf{p})$, the optimal solution is achieved at $k=\arg \max _{k^{\prime} \in \mathcal{K}_{m}} r_{m, k^{\prime}}^{n}(\mathbf{p})$, which means that the optimal subchannel allocation scheme is to assign the subchannel to the user that can offer the highest data rates. 


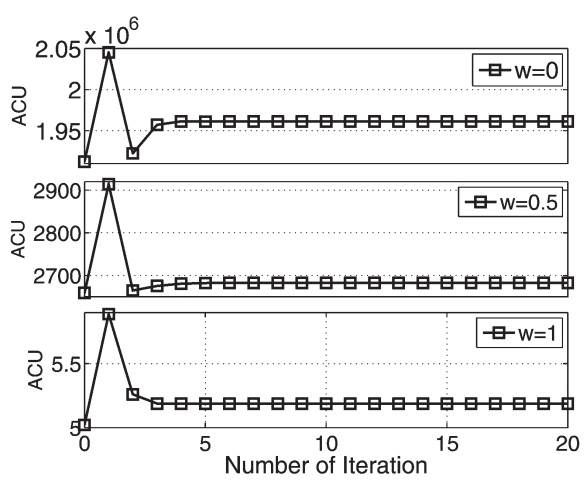

(a)

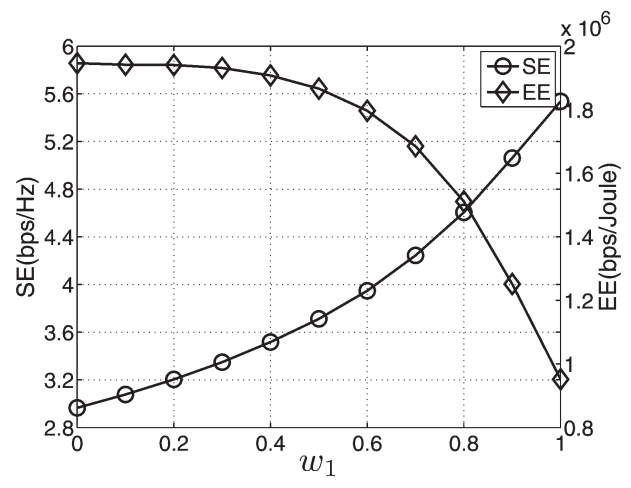

(b)

Fig. 2. (a) The convergence, where $w_{m}=w, K_{m}=30, \forall m \in \mathcal{M}$. (b) The EE and SE of BS 1 , where $w_{3}=w_{5}=w_{7}=0, w_{2}=w_{4}=w_{6}=1$ and $K_{m}=30, \forall m \in \mathcal{M}$.

\section{B. Power Allocation for a Given Subchannel Allocation}

When the subchannel allocation matrix $\mathbf{s}=\left[s_{m, k}^{n}\right]_{M \times K_{m} \times N}$ for all $m \in \mathcal{M}$ is given, the problem (3) is simplified into a power allocation problem:

$$
\max _{\left\{p_{m}^{n}\right\}} \sum_{m \in \mathcal{M}} F_{m}(\mathbf{s}) \quad \text { s.t. }(3 \mathrm{~b})(3 \mathrm{~d}),
$$

where $F_{m}(\mathbf{s})=\log _{2}\left[\sum_{n \in \mathcal{N}} r_{m, k^{*}(m, n)}^{n}\right]-w_{m} \log _{2}(N B)-(1-$ $\left.w_{m}\right) \log _{2}\left(\xi \sum_{n \in \mathcal{N}} p_{m}^{n}+p_{c}\right)$ and $k^{*}(m, n)$ denotes the user to which the subchannel $n$ is allocated in $\mathrm{BS} m$.

The problem (7) is not a convex-problem due to the nonconvexity of the objective function. As it is computationally hard to find the global optimal solution for a non-convex problem, we devise a low-complexity heuristic power allocation algorithm relying on the Lagrangian dual method [11]. The Lagrangian function for the problem (7) can be derived as

$$
L(\lambda)=\sum_{m \in \mathcal{M}}\left[F_{m}(\mathbf{s})+\lambda_{m}\left(P_{m}^{\max }-\sum_{n \in \mathcal{N}} p_{m}^{n}\right)\right],
$$

where $\lambda=\left[\lambda_{1}, \cdots, \lambda_{m}, \cdots, \lambda_{M}\right]$ is the dual vector composed of nonnegative Lagrangian multipliers with respect to constraint (3b).

According to the Karush-Kuhn-Tucker (KKT) conditions, upon taking derivative of the Eq. (8) with respect to $p_{m}^{n}$, it can be derived that the solution of the problem (7) must satisfy

$$
p_{m}^{n}=\left[\frac{1}{\beta_{m}^{S E}+\beta_{m}^{E E}+t_{m}^{n}}-\frac{I_{m, k^{*}(m, n)}^{n}+\sigma^{2}}{g_{m, k^{*}(m, n)}^{n}}\right]^{+},
$$

where $[x]^{+}=\max \{0, x\}, \quad \beta_{m}^{S E}=\eta_{m}^{S E} \lambda_{m}(\ln 2)^{2}, \quad \beta_{m}^{E E}=$ $\eta_{m}^{E E} \frac{\left(1-w_{m}\right) \xi(\ln 2)}{B} 1$ and

$$
t_{m}^{n}=\sum_{j \in \mathcal{M} \backslash\{m\}} R_{m} E_{j, k}^{n},
$$

in which $E_{j, k}^{n}=\frac{p_{j}^{n} g_{j, k^{*}(j, n)}^{n} g_{m, k^{*}(j, n)}^{n}}{R_{j}\left(I_{j, k^{*}(j, n)}^{n}+\sigma^{2}\right)^{2}\left(1+\operatorname{SINR}_{j, k^{*}(j, n)}^{n}\right)}$.

Note that the Eq. (9) is similar to the power allocation scheme of the Modified Iterative Water-Filling (MIWF) algorithm [1].

\footnotetext{
${ }^{1}$ In practical implementation, BS's $\mathrm{EE}$ and $\mathrm{SE}$ performance in current iteration (i.e., $\eta_{m}^{S E}$ in $\beta_{m}^{S E}$ and $\eta_{m}^{E E}$ in $\beta_{m}^{E E}$ ) can be approximated by a linear predication scheme, based on EE and SE performance achieved in previous iterations.
}

However, there are significant differences. The "water-level" of the proposed power allocation scheme is dependent not only on $t_{m}^{n}$, but also on the EE and SE performance of the corresponding $\mathrm{BS}$ (i.e., $\beta_{m}^{S E}$ and $\beta_{m}^{E E}$ ). Therefore, the proposed algorithm's essence of the EE-SE joint optimization has been imbedded in (9).

Finally, based on the subgradient method, the dual variables can be updated until convergence is achieved, according to

$$
\lambda_{m}^{(i+1)}=\left[\lambda_{m}^{(i)}-\Gamma_{m}^{(i)}\left(P_{m}^{\max }-\sum_{n \in \mathcal{N}} p_{m}^{n}\right)\right]^{+}, \forall m \in \mathcal{M},
$$

where $\Gamma_{m}^{(i)}$ is the step size of the $i$ th iteration.

\section{Iterative Subchannel and Power Allocation Algorithm}

The details of the proposed iterative subchannel and power allocation (ISP) algorithm are shown in Fig. 1. The ISP algorithm can be implemented at each BS, but requires BSs' coordination for exchanging the information of $E_{j, k}^{n}$ in order to calculate $t_{m}^{n}$ in Eq. (9), and each BS will send $(M-1) N$ signaling information concerning $E_{j, k}^{n}$ during each iteration. Let us denote $I_{\max }$ as the maximum number of iterations of the outer loop in the ISP algorithm and $K=\sum_{m \in \mathcal{M}} K_{m}$, then the computation complexity of the ISP algorithm is characterized by $\mathcal{O}\left[I_{\max }\left(N K+M N /\left(1 / \varepsilon^{2}\right)\right)\right]$, which is a polynomial complexity and facilitates the practical implementation ${ }^{2}$.

\section{Simulation And Numerical Analysis}

The performance of the ISP algorithm is evaluated using Monte Carlo method. We consider a 49-cell topology similar to the cellular OFDMA system in [1]. Note that the central cluster of 7 cells is coordinated, while the remaining BSs are treated as the source of uncoordinated other-cell interference. Assume that 60 subchannels are available, and each occupies a bandwidth of $200 \mathrm{KHz}$. The Hata urban propagation model is used for characterizing the pass-loss effect. Each subchannel experiences independent identically distributed Rayleigh fading

\footnotetext{
${ }^{2}$ Note that the proposed ISP is a heuristic algorithm because of the separate optimization of the power and subchannel allocations, as well as of the iterative procedure for updating their respective solutions. Therefore, it does not guarantee the globally optimal solution.
} 


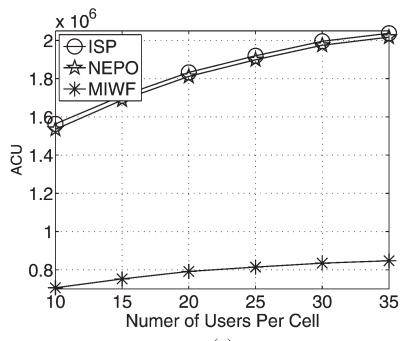

(a)

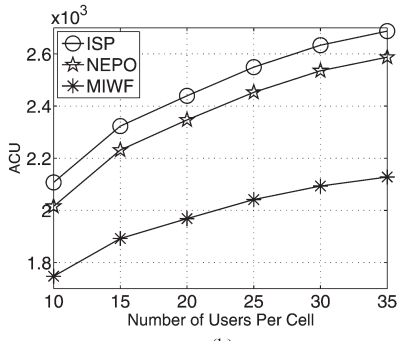

(b)

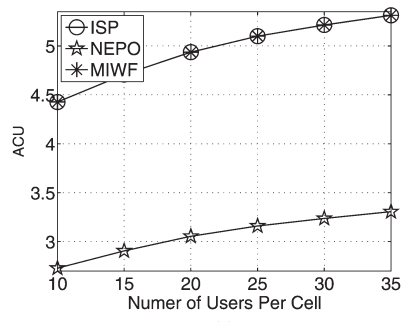

(c)

Fig. 3. The ACU, when (a) $w_{m}=0, \forall m \in \mathcal{M}$. (b) $w_{m}=0.5, \forall m \in \mathcal{M}$. (c) $w_{m}=1, \forall m \in \mathcal{M}$.

with unit average power gain. For all BSs, $P_{m}^{\max }, p_{c}$ and $\xi$ are set to $20 \mathrm{~W}, 10 \mathrm{~W}, 2.63$, respectively.

The convergence of the ISP algorithm in terms of the average cell utility (ACU, is the average value of the utility of the 7 coordinated cells) is shown in Fig. 2(a). It can be observed that the ACU converges to stable value with about 5 iterations no matter what specific value $w$ has, which means that the convergence of the ISP algorithm is guaranteed.

The impact of the preference factor $w$ on the corresponding $\mathrm{EE}$ and SE is shown in Fig. 2(b). The performance of the BS in the center of the network, i.e., BS 1 , is chosen as an example. It can be seen that the SE increases while the EE decreases with the increase of $w_{1}$. This is consistent with the intuition, because a larger value of $w_{1}$ indicates that higher priority is given to the SE optimization. From Fig. 2(b), the effectiveness of the EST metric in the multi-cell network scenario is verified, because it can capture the tradeoff relation between EE and SE. Hence, the operator can adapt the preference factor $w_{m}$ of each BS to balance their EE and SE performance.

The performance of the proposed ISP algorithm in terms of ACU is evaluated in Fig. 3, compared with two other resource allocation algorithms. Both of them implement the same subchannel allocation algorithm proposed in this letter, but with two different power allocation algorithms: one is the MIWF algorithm [1] that is considered here as an example of the SEbased algorithm, and the other is the non-cooperative energyefficient power optimization (NEPO) algorithm [3], which is included as an example of the EE-based algorithm. In Fig. 3, different values of $w_{m}$ mean different priorities for EE or SE optimization. More specifically, $w_{m}=0$ indicates the EEoptimal optimization mode, $w_{m}=1$ represents the SE-optimal optimization mode, while $w_{m}=0.5$ characterizes the EE-SE tradeoff optimization mode. It can be seen that the ACU of all three algorithms attain higher values with the increase of the user number regardless of $w$. The performance gain is owing to the multi-user diversity. Additionally, from Fig. 3(a) and (c), we can observe that when $w=0$, the ACU of ISP algorithm is slight higher than that of the NEPO algorithm, ${ }^{3}$ and almost the same as that of the MIWF algorithm when $w=1$. This means that the proposed ISP algorithm is capable of achieving a comparable performance with the existing EE-optimal or SEoptimal based algorithms, when the optimization objective is set to the mode of EE-optimal $\left(w_{m}=0\right)$ or SE-optimal $\left(w_{m}=\right.$ 1). Furthermore, when $w=0.5$, the ACU of the ISP algorithm is

\footnotetext{
${ }^{3}$ Since the NEPO algorithm is a non-cooperative game-based algorithm without information exchange between BSs, the performance gain of the ISP algorithm over the NEPO algorithm when $w=0$ is attributed partly to ISP's coordination mechanism between the BSs, which is achieved at the cost of moderately extra signaling load.
}

significantly higher than that of the MIWF or NEPO algorithm, just as Fig. 3(b) shows. This performance gain substantiates the advantage of the ISP algorithm in terms of 1) the flexibility to adjust radio resource allocation for satisfying BSs' divergent requirements on SE and EE priorities and 2) to make joint optimization of both relevant metrics.

\section{CONCLUSION}

In this letter, we investigate the resource allocation in multicell OFDMA networks in order to jointly optimize the EE and SE performance. The joint optimization problem is formulated based on a novel EST metric, and is solved relying on the Lagrangian dual method. An ISP algorithm is presented for the resource allocation, which allocates the subchannel and power iteratively. Simulation results demonstrate that the proposed ISP algorithm has significant advantages and flexility in EE and SE optimization compared with the existing MIWF and NEPO algorithms. In our future work, we will investigate the performance gap, if any, between the proposed ISP algorithm and the optimal algorithm.

\section{REFERENCES}

[1] L. Venturino, N. Prasad, and X. Wang, "Coordinated scheduling and power allocation in downlink multicell OFDMA networks," IEEE Trans. Veh. Technol., vol. 58, no. 6, pp. 2835-2848, Jul. 2009.

[2] K. Cheung, S. Yang, and L. Hanzo, "Achieving maximum energyefficiency in multi-relay OFDMA cellular networks: A fractional programming approach," IEEE Trans. Commun., vol. 61, no. 7, pp. 27462757, Jul. 2013.

[3] G. Miao, N. Himayat, G. Li, and S. Talwar, "Distributed interferenceaware energy-efficient power optimization," IEEE Trans. Wireless Commun., vol. 10, no. 4, pp. 1323-1333, Apr. 2011.

[4] G. Miao, N. Himayat, G. Li, and S. Talwar, "Low-complexity energyefficient scheduling for uplink OFDMA," IEEE Trans. Commun., vol. 60, no. 1, pp. 112-120, Jan. 2012.

[5] H. Zhang et al., "Resource allocation in spectrum-sharing OFDMA femtocells with heterogeneous services," IEEE Trans. Commun., vol. 62, no. 7, pp. 2366-2377, Jul. 2014.

[6] C. Xiong, G. Li, S. Zhang, Y. Chen, and S. Xu, "Energy- and spectralefficiency tradeoff in downlink OFDMA networks," IEEE Trans. Wireless Commun., vol. 10, no. 11, pp. 3874-3886, Nov. 2011.

[7] Y. Li, M. Sheng, C. Yang, and X. Wang, "Energy efficiency and spectral efficiency tradeoff in interference-limited wireless networks," IEEE Commun. Lett., vol. 17, no. 10, pp. 1924-1927, Oct. 2013.

[8] C. He, B. Sheng, P. Zhu, and X. You, "Energy efficiency and spectral efficiency tradeoff in downlink distributed antenna systems," IEEE Wireless Commun. Lett., vol. 1, no. 3, pp. 153-156, Jun. 2012.

[9] C. He, B. Sheng, P. Zhu, X. You, and G. Li, "Energy- and spectralefficiency tradeoff for distributed antenna systems with proportional fairness," IEEE J. Sel. Areas Commun., vol. 31, no. 5, pp. 894-902, May 2013.

[10] L. Deng et al., "A unified energy efficiency and spectral efficiency tradeoff metric in wireless networks," IEEE Commun. Lett., vol. 17, no. 1, pp. 5558, Jan. 2013.

[11] D. Ng, E. Lo, and R. Schober, "Energy-efficient resource allocation in OFDMA systems with hybrid energy harvesting base station," IEEE Trans. Wireless Commun., vol. 12, no. 7, pp. 3412-3427, Jul. 2013. 